\section{$\underset{\substack{\text { hommes } \\ \text { \& migrations }}}{ }$}

\section{Hommes \& migrations}

Revue française de référence sur les dynamiques

migratoires

$1312 \mid 2015$

Diasporas iraniennes

\title{
Accueillir des rescapés
}

Le regard des travailleurs sociaux

\section{Orly Knafo et Claudine Salamon}

\section{OpenEdition \\ 1 Journals}

\section{Édition électronique}

URL : http://journals.openedition.org/hommesmigrations/3517

DOI : 10.4000/hommesmigrations.3517

ISSN : 2262-3353

\section{Éditeur}

Musée national de l'histoire de l'immigration

\section{Édition imprimée}

Date de publication : 1 octobre 2015

Pagination : 133

ISBN : 978-2-919040-33-9

ISSN : 1142-852X

Référence électronique

Orly Knafo et Claudine Salamon, « Accueillir des rescapés », Hommes \& migrations [En ligne], 1312 |

2015, mis en ligne le 31 mai 2016, consulté le 14 septembre 2020. URL : http://

journals.openedition.org/hommesmigrations/3517 


\section{REPÉRAGE}

\section{ACCUEILLIR DES RESCAPÉS LE REGARD DES TRAVAILLEURS SOCIAUX}

par ORLY KNAFO, chef du Service social familial (Fondation Casip-Cojasor), et CLAUDINE SALAMON, directrice du Service social (Fondation Casip-Cojasor).

I s'agit de deux situations de réfugiés juifs origi-

naires d'Europe centrale et orientale, survivants des camps nazis. La première, E. U., femme seule, se présente au Cojasor en novembre 1945 et sera suivie jusqu'en août 1948. La seconde, la famille S., se compose d'un couple de rescapés et d'une petite fille née en 1946. La famille sera suivie de 1948 à 1952.

Dans ces deux cas, les assistantes sociales apportent des réponses à chaque demande dans les domaines concernant les besoins essentiels de la vie quotidienne. C'est ainsi que dès le premier rendez-vous, la discussion entre l'assistante et E. U. porte surtout sur la question du travail. Bien que celle-ci “désire apprendre un métier" et le français, l'assistante lui demande de chercher d'abord du travail et l'oriente vers son bureau de placement et l'Alliance française pour les cours de langue. Elle lui octroie aussi une aide au logement, une aide alimentaire et vestimentaire, le temps qu'E. $U$. soit indépendante. Pour la famille $S$, le mari souffrant ne peut pas travailler de manière continue et intensive. Les sommes versées par les assurances sociales ne permettent toutefois pas de couvrir toutes les dépenses du foyer, d'autant que les revenus de la mère sont irréguliers et insuffisants. Le Cojasor leur attribue alors une aide financière régulière, complétée de dons de vêtements, et oriente Mme S. vers des organismes spécialisés dans le reclassement professionnel (Copij et
Cose pour un placement, ORT pour l'achat d'une machine à coudre). Nous constatons une différence dans la forme des dossiers $U$. et S., qui révèle une évolution des pratiques professionnelles. Le dossier le plus tardif comporte une fiche familiale plus élaborée, avec notamment une case sur le motif de cessation du suivi, des "résumés de transmission" entre assistantes et un "résumé de clôture" en réalité très détaillé. Figure aussi un rapport du fichier central de l'American Joint Distribution Committee de 1951, qui informe l'assistante sur les institutions juives ayant aussi secouru la famille. Les méthodes de travail se perfectionnent donc, le dossier S. donne des informations plus précises, non limitées aux aspects matériels mais portant aussi sur les relations familiales et l'histoire générale de la famille. De plus, il se termine plus clairement.

Dans le dossier U., il est écrit: "Fait bonne impression". Dans le dossier S., nous lisons sur le père de famille : "Il est aigri et vieilli", et sur Mme S. qu'elle est "jeune et courageuse". Dans les deux dossiers, les notes des assistantes sociales comportent des observations qui sont leurs impressions personnelles, des jugements de valeur portés sur les personnes suivies. Ce type d'écrit révèle que la distance professionnelle et la neutralité, principes essentiels dans le travail social d'aujourd'hui, n'avaient pas encore été enseignées aux travailleurs sociaux de cette époque. 